Horizons philosophiques

\title{
Le monde de la musique et la musique comme monde selon Deleuze
}

\section{Vincent Jacques}

Volume 16, numéro 1, automne 2005

Raisonner la musique

URI : https://id.erudit.org/iderudit/801302ar

DOI : https://doi.org/10.7202/801302ar

Aller au sommaire du numéro

Éditeur(s)

Collège Édouard-Montpetit

ISSN

1181-9227 (imprimé)

1920-2954 (numérique)

Découvrir la revue

Citer cet article

Jacques, V. (2005). Le monde de la musique et la musique comme monde selon Deleuze. Horizons philosophiques, 16(1), 1-23. https://doi.org/10.7202/801302ar d'utilisation que vous pouvez consulter en ligne.

https://apropos.erudit.org/fr/usagers/politique-dutilisation/ 


\title{
Le monde de la musique et la musique comme monde selon Deleuze
}

\author{
"L'univers, le cosmos est fait de ritournelles : la question de \\ la musique est celle d'une puissance de déterritorialisation \\ qui traverse la nature, les animaux, les éléments et les \\ déserts non moins que l'homme" \\ Mille Plateaux, p. 380
}

La nature comme musique. Excluons d'emblée toute interprétation métaphorique : Deleuze a toujours déclaré que son faire philosophique était conceptuel et le thème de la «musique» ne fait pas exception. La difficulté, pour qui veut saisir ce rapport philosophie/musique chez Deleuze, est que celui-ci se joue à plusieurs niveaux. Tout d'abord, il y a les quelques pages dédiées à la musique comme telle, considérations conceptuelles qui, par nature, engagent déjà tout le système. Mais à un autre niveau, la musique offre aussi un matériau brut à la création conceptuelle, comme cela est tout particulièrement sensible dans Mille Plateaux. Deleuze esquisse ainsi à la fois une esthétique musicale, et emprunte des concepts, des notions à des musiciens (Boulez, Messiaen, Stockhausen) pour bâtir son système philosophique. Disons aussi que ses préoccupations philosophiques - combattre le système de la représentation, saisir une pensée émancipée des formes trop strictes et de voies tracées a priori, trouvent écho dans l'effort de nombreux compositeurs contemporains cherchant à construire une musique émancipée des déterminismes trop stricts de la tradition : comment sentir le chaos (hasard)? Deleuze émit naguère l'idée que le Nietzsche musicien n'est pas tant celui qui a composé, mais celui qui a fait passer la musique dans son œuvre écrite ${ }^{1}$. Disons à notre tour que le Deleuze "musicien-musicologue» n'est pas seulement celui qui a écrit explicitement sur la musique, mais celui qui a composé une œuvre philosophique conceptuelle avec une intuition particulièrement musicale, Pli selon pli. 
Nous commencerons par exposer longuement le monde de la musique selon Deleuze pour bien saisir l'importance de sa critique de la représentation-médiation et sa logique existentielle de la sensation élaborée en grande partie avec certaines idées et intuitions qui émanent de certains compositeurs contemporains. Nous essaierons de montrer que l'esthétique musicale deleuzienne, même quand elle ne parle que de musique, engage tout le système, et notamment ses nuances existentielles. Car dans la deuxième partie, sur la musique comme monde, il sera aussi question en définitive des nuances existentielles d'un être-au-monde qui est au monde en l'exprimant selon des modes rythmiques, mélodiques, passion de la ritournelle. Le Dasein heideggerien, flux et reflux, jeté dans le monde et, en quelque sorte, se retirant de cette projection dans le monde outillé des étants, ouvre un espace d'attente et d'écoute de l'être; le "sujet" deleuzien, lui, s'extrait des rengaines répétitives et sclérosantes des habitudes pour, rythme et mélodie, en arriver à se dissoudre, à filer avec le monde en le faisant filer en retour, fuser avec le monde, s'y couler et le faire couler, sorte d'extase existentielle vers laquelle tend toute l'œuvre : devenir-imperceptible. Fusion mondaine, ligne de fuite, accroissement de la sensation, être-la-sensation-monde, toute cette série de notions fondamentales de la philosophie deleuzienne, est, comme nous proposons de le démontrer, impensable sans une certaine pensée de la musique.

Notre exposé se divisera donc en deux parties : 1- nous dégagerons les grandes lignes de la pensée deleuzienne de la musique, esthétique préoccupée par les problèmes de la forme, du temps, de la sensation, des forces et de la déterritorialisation, dégageant ainsi une visée critique et une visée existentielle et $c$ réatrice, 2- nous analyserons ensuite l'enchaînement conceptuel exposé dans Mille Plateaux (11. 1837 - De la ritournelle), extraordinaire physique musicale où des personnages rythmiques évoluent dans des paysages mélodiques, devenir-monde d'un entrelacs dynamique de petites ritournelles ouvertes sur la grande où se joue une étonnante symphonie de milieux, de territoires et de déterritorialisations. Physique philosophique à l'intuition musicale, construite avec des notions empruntées à la musique, et posant un problème commun et actuel philosophie-musique : celui de la consistance, tenir-ensemble d'hétérogènes. Avec ses deux chemins parcourant l'esthétique, la physique et la métaphysique, sillonnons donc un moment la vaste contrée deleuzienne ${ }^{2}$ sous le signe de la musique. 
L'illusion d'Apollon : l'éternité des formes belles, législation aristocratique : «il doit toujours en être ainsi». Dionysos : sensualité et cruauté. L'instabilité des choses pourrait être interprétée comme la jouissance d'une force qui engendre et détruit, comme création perpétuelle

Nietzsche, Fragment posthume, 1885-1886 (XVI, B 1049)

L'esthétique musicale deleuzienne se comprend à l'aune de sa critique radicale de la représentation. C'est dans Différence et répétition que Deleuze expose le plus en détail cette critique : la pensée de la représentation est incapable de «penser la différence en elle-même»: C'est avec Platon que commence le système de la représentation basé sur le primat de l'identité qui voilera bien vite ses soubassements moraux pour donner naissance à cette créature à quatre têtes qu'est la médiation. Identité, analogie, opposition et ressemblance sont les quatre organes du système de la représentation comme raison, longue erreur qui traverse toute l'histoire de la philosophie. Cette critique, pourtant, n'est que le corollaire de ce qui intéresse vraiment Deleuze, à savoir dégager ce qu'il y a sous cette représentation trop fixe, trop rigide, trop creuse : le monde grouillant de la différence et de la répétition, peuplé d'individuations impersonnelles et de singularités pré-individuelles, monde des pures intensités. Arriver à saisir le vrai mouvement de la vie, faire - un avec elle, au-delà des blocages et des concrétions que secrète trop spontanément l'homme, ce "mammifère à frein" (Michaux), voilà le travail qu'assigne Deleuze à la philosophie.

L'esthétique deleuzienne, donc, ne fera pas exception à cette règle. Elle traquera les formes à tous les niveaux : malheur de toute détermination ${ }^{3}$. Notons tout d'abord que rien n'est plus étranger à Deleuze que «l'art pour l'art». L'art n'est pas une fin en soi, il n'est qu'un moyen pour tracer des lignes de vie, c'est-à-dire faire-un avec des devenirs bien réels qui sont ceux du monde, mais qui ne préexistent pas à une ligne de fuite active ${ }^{4}$. La fuite n'est pas une fuite dans l'art, mais un processus de déterritorialisation qui emporte tout et, qui partant de l'art, ne doit pas y aboutir. L'art n'a donc qu'un statut privilégié de catalyseur, de révélateur ou d'accélérateur de devenirs, agencement privilégié pour nous libérer des trop stricts domaines cloisonnés qui limitent et étreignent nos sensations, notre perception et notre pensée. Puisque l'art n'est donc pas une fin en soi, il est néfaste de le couper des processus vitaux qui l'engendrent et qu'il 
produit. À ce refus d'un art autonome se mirant lui-même tout imbu de son illusoire suffisance, vient logiquement s'ajouter chez Deleuze le rejet de l'Art comme système structuré et hiérarchisé, où à une répartition des sens répond les domaines d'arts bien distincts : "nous ne croyons nullement à un système des beaux-arts, mais à des problèmes très différents qui trouvent leurs solutions dans des arts hétérogènes ${ }^{\text {». }}$. Pour Deleuze, toute détermination trop stricte a priori, découpant des places et assignant des voies, met au pas et limite la création et par conséquent la réception de l'œuvre. Sages créateurs pour gentils récepteurs - système du divertissement généralisé. À ces disciplines des sens et des arts est opposée une multisensorialité vers laquelle devrait tendre toute œuvre ${ }^{6}$. Rien de plus étranger, non plus, donc, à cette esthétique deleuzienne, que la recherche d'une musique «pure».

Dans Logique du sens, Deleuze démontre que la peinture de Bacon tend à dépasser le système figuratif représentation/vision, pour atteindre à une vision haptique7, au-delà de la médiation et de la répartition des sens, mettant en branle le toucher puis toute la sensibilité pour atteindre directement le système nerveux grâce à une ligne de fuite emportant le sentant et le senti. Comme l'écrit M.Buydens, il s'agit de ufusionner avec les forces qui peuplent les choses ${ }^{8}$ ", d'atteindre un rythme ou une pulsation fondamentale. La médiation m'empêche d'être pleinement au monde, de fuir avec lui, avec la vie, de fusionner avec elle, médiation qui me met dans cette position contre laquelle Nietzsche n'avait pas de mots trop durs : celle de juger la vie.

Si le système des beaux-arts est rejeté et la multisensorialité souhaitée, Deleuze ne rejette pas pourtant les arts particuliers. Tout n'est que question de rapport, de proportion. C'est ainsi qu'il oppose peinture et musique, et compare leur pouvoir de déterritorialisation respectif : "Dès lors, la seule manière de "ranger" les deux problèmes, de la peinture à la musique, c'est de prendre un critère extrinsèque à la fiction des beaux-arts, c'est de comparer les forces de déterritorialisation dans les deux cas. Or il semble que la musique ait une force déterritorialisante beaucoup plus grande, beaucoup plus intense et collective à la fois ${ }^{\prime}$ \%. Ce pouvoir de déterritorialisation supérieur s'explique facilement : la musique travaille avec une matière fluide, désincarnée, tisse l'immatériel lui-même, compose avec le temps et les ondes sonores. Le musicien, ici, tend à se faire l'opérateur de pures intensités, tandis que le peintre, lui, se débat dans la 
matière brute, toile, pâte, truelle, pigment, liant. De son étreinte avec la matière, le peintre fera jaillir les forces entrelacées de la chair du monde directement sur nos sens. Là où le peintre nous met en contact direct avec toute la «pesanteur» du monde, le musicien nous branche directement sur son "apesanteur": deveniroiseaux de Mozart. Nous pouvons suivre ici l'hypothèse de Buydens ${ }^{10}$ : si nous évaluons la peinture et la musique par rapport à leur aptitude à échapper ou à "faire fuir» les formes, la peinture tend à fuir "par le bas", dans le mélange des corps et dans la matière brute, tandis que la musique fuit "par le haut», ascension vers l'immatérialité de cette énergie pure qui meut ces corps, sphère des intensités libérées (bipolarité peinture/matière, musique/force).

Ajoutons finalement un point pour conclure cette comparaison musique/peinture. II est certain que l'affinité de Deleuze pour la musique peut aussi s'expliquer par le fait que celle-ci est l'art nonreprésentatif par excellence dans nos sociétés fondées sur la préséance de la vision ${ }^{11}$. Saint-Thomas, patron d'Occident, vu?.... Vision, ou primat diffus de la représentation médiatrice dans la triade grossière, "ce qu'il y a" à voir, "représentation" donnant ce «il y a» à voir, sujet voyant le «il y a" de la représentation. D'où l'injonction deleuzienne de ne pas illustrer, interpréter et reproduire les choses, mais plutôt de tracer des lignes de vie à même le réel : «faites la carte et pas le calque ${ }^{12}$ ». Là où l'image rassure, d'ailleurs que de mauvaises interprétations d'œuvres musicales où l'on traduit ce que la musique voudrait montrer - triste destin des symphonies de Beethoven sur lesquelles on plaque impunément des images - la musique fait peur, est plus ambiguë, et est plus propice du coup à mobiliser et à fasciner les foules : «la musique, tambours, trompettes, entraîne les peuples et les armées, dans une course qui peut aller jusqu'à l'abîme, beaucoup plus que ne le font les étendards et les drapeaux, qui sont des tableaux, des moyens de classification ou de ralliement ${ }^{13}$ ".

Passons maintenant de cette comparaison musique/peinture à la seule musique. L'esthétique musicale deleuzienne est fondée sur la distinction temps pulsé / temps non pulsé proposée par Boulez ${ }^{14}$. Le temps pulsé est un temps subordonné à la métrique d'un quadrillage : «pour autant que les coupures y sont déterminables, c'est-à-dire de type rationnel (premier aspect du continu), et les mesures, régulières ou non, déterminées comme des grandeurs entre coupures ${ }^{15}$ ". Contrairement à ce temps soumis à la pulsation et dominant la 
tradition musicale occidentale, le temps non pulsé, lui, «ne se réfère plus à la chronométrie que de façon globale : les coupures y sont indéterminées, de type irrationnel, et les mesures sont remplacées par les distances et voisinages indécomposables qui expriment la densité ou la rareté de ce qui apparaît (répartition statistique d'événements) ${ }^{16}$ ». Ces deux pôles distincts renvoient aux deux types d'espaces exposés dans Mille Plateaux, eux-mêmes corrélatifs des ces deux grandes catégories d'être-au-monde que sont le nomadisme et le sédentarisme. D'un côté nous retrouvons l'espace strié du sédentaire, quadrillé et hiérarchisé, divisé en lots, soumis à la cadence et au principe d'identité, partagé a priori par le grand Signifiant. De l'autre nous avons l'espace lisse, exempt de toute métrique, espace fluide peuplé de lignes et de vitesses (intensités), espace nomade ouvert à toute connexion, sans buts ni fin. "Remplir un espace (espace-temps lisse) n'est pas partager l'espace (espace-temps strié)" dira Deleuze à la suite de la formule boulezienne "occuper sans compter" (n'est pas compter pour occuper). On aura compris que le pôle nomade est valorisé par rapport au pôle sédentaire qui a la préséance dans nos sociétés : il s'agit toujours ici de la recherche des libres devenirs au-delà de la gangue rigidifiée des formes et de la représentation, et en définitive de l'intuition spinoziste : omnis determinatio est negatio. Le systèmepoints de l'espace-temps strié soumet la ligne, la coupe, la détermine comme dimension métrique. L'espace temps lisse, lui, laisse vagabonder les lignes, devenirs qui remplissent l'espace et le temps selon un mode d'action libre (vitesse), où le point est subordonné au procès, contrairement à la détermination pulsatrice - d'un point à l'autre, où le procès est subordonné au point.

Toute l'œuvre de Deleuze, et tout particulièrement Mille Plateaux, machination nomade tout azimut, scrute avec extrême minutie le devenir des lignes ${ }^{17}$ (séries), leurs rencontres et ce qui en fulgure, prise fulgurante qui «a l'inconvénient de résister à long terme, de ne pouvoir se déprendre ${ }^{18}$ ". Voilà le problème : avec le temps, nous sommes littéralement ensevelis par toutes ces formes fossiles, scories et autres cadavres rigidifiés qui furent et sont encore nos idées, nos passions, nos affects. L'homme, donc, par nature habitué à "freiner», est enseveli de surcroît par toutes les froides scories de son histoire et, aujourd'hui, arrimé dans cette excroissance mondiale et totalisante du "plus froid des monstres froids", machination planétaire et sédentaire, qui tout absorbé dans son babillage sur les flux et la 
mobilité, ignore et bloque d'autant plus tout vrai mouvement, flux et lignes vitales ${ }^{19}$.

Précisons tout de suite que cette distinction strié/lisse est une de droit, ce qui n'est nullement contradictoire avec la réalité des mélanges de fait2o : en effet le plus strié laisse toujours échapper du lisse tandis que le lisse finit toujours par secréter du strié. Ainsi, pour en revenir au temps musical, il y a "échange entre les deux fonctions de temporalisation, ne serait-ce qu'au sens où une répartition homogène dans un temps strié donne l'impression d'un temps lisse, alors qu'une distribution très inégale en temps lisse introduit des directions qui évoquent un temps strié, par densification ou accumulation de voisinage ${ }^{21}$ ». Boulez parle d'un temps lisse, non pulsé ou d'un temps amorphe, temps musical qui ne se rapporte au temps chronométrique que sous l'aspect de la globalité (durée de l'œuvre) tandis que le temps strié est celui du chronomètre, celui qu'on découpe, celui qu'on périodise. Temps pulsé et temps non pulsé sont donc deux pôles, deux tendances que nous retrouverons dans toute œurre musicale, selon diverses proportions. Ces deux pôles temporels sont aussi deux "matières" que travaille le compositeur, et selon le pôle qu'il privilégie, il ne se produira pas dans son œuvre les mêmes choses.

Notons au passage que cette distinction boulezienne fait écho à la distinction deleuzienne entre Aïon et Chronos introduite dans Logique du sens et reprise dans Mille plateaux : "Aïon est le temps indéfini de l'événement, la ligne flottante qui ne connaît que les vitesses (...) et Chronos, au contraire, le temps de la mesure, qui fixe les choses et les personnes, développe une forme et détermine un sujet22». Voilà un des enjeux fondamentaux de l'esthétique musicale deleuzienne : accéder à cette ligne flottante, à ce monde grouillant bloqué et cadré par la forme, accentuer la variation continue du temps et des ondes sonores au détriment de toute détermination métrique constituant une trame d'a priori appelant à la récognition identitaire. Tout est ici question de tendance vers l'un des deux pôles, car la musique, plus que toute autre forme d'art, est de manière générale opposée à l'émergence en elle de formes qui appellent trop fortement une récognition identitaire : «la musique a toujours eu cet objet : des individuations sans identité, qui constituent les "êtres musicaux"23". Mais d'un point de vue intrinsèque à la composition musicale elle-même, le fait de tendre vers tel ou tel pôle, temps pulsé ou temps 
non pulsé, pose le problème du milieu où, selon la tendance, ne se développeront pas les mêmes événements musicaux. Au temps pulsé qui domine jusqu'à aujourd'hui le monde de la musique occidentale correspond "une musique formelle et fonctionnelle fondée sur les valeurs", une musique posant le «jeu organique de l'identique et du varié", tandis qu'avec la trame d'un temps non pulsé se crée une "musique flottante et machinique qui n'a plus que des vitesses ou des différences dynamiques", déployant le " jeu fonctionnel de la différence et de la répétition $24 »$. La musique flottante n'est plus peuplée de formes se développant selon un principe identitaire qui se laisse varier pour mieux se laisser retrouver, mais, tout comme la meute nomade se répartissant selon les aléas de son propre entrain dans un espace ouvert, fait se mouvoir une variation continue faite de flux sonores, de densités, d'intensités, jeu différentiel non repérable 25 . Deux tendances, donc, qui en amont déterminent le compositeur à privilégier soit la forme et son développement, soit le vagabondage du flux sonore; soit les relations structurales, soit les forces et les matériaux au détriment d'une conceptualisation et d'une armature identitaire et sédentaire. En aval, l'auditeur, lui aussi, n'est plus dans la même position, qu'il soit le sujet médiatisé opérant la récognition des formes et réfléchissant l'entièreté de la structure grâce à sa mémoire, où qu'au contraire il soit ce point de capiton mobile irrigation nerveuse à vif - qui se conjugue sans médiation au flux vagabond sonore, liant son propre devenir à celui de la musique, sans mémoire et sans attente 26 , ouvert dans son innocence à toutes les brusques irruptions de l'imprévu. Insistons sur ce point : cette esthétique de l'immédiateté ne peut absolument pas être séparée d'un ethos du vagabondage, nécessité des lignes de fuites, sous peine de se confondre avec la fragmentation des petites jouissances propre à "l'art» du Capital : esthétique industrielle, qui, pour pimenter son inlassable monotonie, parsème ses platitudes de petites "surprises" pour une masse amorphe.

Qu'il n'y ait pas ici d'ambiguïtés : Deleuze ne condamne pas la musique fonctionnelle, il pointe plutôt un nouveau paradigme de la composition musicale, posé par Boulez, mais aussi appliqué de différentes manières par Stockhausen, Berio, Xenakis, nouvelle esthétique qui rencontre une forte résonance avec ses préoccupations philosophiques. On ne peut plus écrire la philosophie comme Platon, ce qui n'empêche pas qu'on lise toujours Platon aujourd'hui en y trouvant une actualité, dira-t-il à propos de l'écriture philosophique; 
a fortiori on peut tout autant dire que Stockhausen n'annule pas Monteverdi27. La musique, comme toute forme de pensée, doit se défaire de toutes gangues inertes héritées du passé, hier formes encore vivantes aujourd'hui déjà froides, pour s'accorder à la tonalité de son époque, et mieux encore atteindre à cet inactuel, à cet intempestif tourné contre l'époque, à celle de lieux communs qui fait de toutes les nouveautés d'hier et d'aujourd'hui des banalités ronflantes et réconfortantes. Esprit du temps critique, qui, selon Deleuze, n'a plus besoin des formes strictes du système de la représentation, abstractions éthérées d'une trop longue méprise morale, et doit donc les combattre pour atteindre plus directement le monde fluant des «individuations sans identités».

De cette esthétique musicale indexée sur un temps non pulsé qui libère le flux sonore de son carcan formel - casser la forme et ses gentilles variations, vous laisserez échapper des nuées de vitesses et le cri nu des matériaux ${ }^{28}$ - et qui, en laissant jaillir forces et matériaux sonores, se branche directement sur les nerfs et emporte l'auditeur, on peut dire qu'il s'agit d'une part d'une esthétique de l'immédiateté et de l'acuité de la sensation et d'autre part d'un ethos esthétique du vagabondage : faire-un de la sensation sentant-senti, faire-un d'une conjonction à un pur devenir à lui-même sa propre finalité : double facette d'un être-au-monde esthétique vitaliste, dont le but ultime est d'amener la vie à une puissance supérieure.

Car à cette musique flottante est aussi assignée une autre tâche, celle d'élargir la perception. "C'est que le problème de l'art, le problème relatif à la création, est celui de la perception, et non pas de la mémoire : la musique est pure présence et réclame un élargissement de la perception jusqu'aux limites de l'univers 29 ". Élargir la perception, donc, grâce au médium de l'art, élaboration d'un matériau qui rend visibles les forces invisibles de la matière pour la peinture, élaboration d'un matériau qui rend audibles, perceptibles des forces inaudibles, imperceptibles pour la musique, "rendre la durée sonore", tout comme la philosophie élabore un matériau qui présente, expose, rend pensables des forces autrement impensables. L'émancipation souhaitée et appelée de la forme ne signifie par ailleurs nullement une injonction au laisser-aller de l'indétermination - attente béate et passive d'une miraculeuse épiphanie, rendre perceptibles des forces autrement imperceptibles requiert au contraire un dispositif très travaillé, seul capable de révéler la présence de ce qui, normalement, se dérobe à notre perception 30 . Beaucoup de 
travail et une habilité technique sans failles sont nécessaires pour atteindre la sobre épure d'une ligne de fuite. Ces forces ne sont pas là, à prendre dans leur pur écoulement, mais sont à capturer, à enchaîner au devenir d'un constructivisme qui ainsi les révèle, les rend accessibles à la perception ${ }^{31}$. Ce constructivisme se comporte à la fois comme une sorte d'accélérateur, de catalyseur et de révélateur de forces, dispositif capteur de devenirs qui ne préexistent pas comme tels, mais qui se «montrent» une fois pris dans ces petits tourbillons d'hétérogénèse que fabriquent l'art et la pensée. La musique bâtit donc elle aussi des dispositifs filtrant, tel un fluide crible, la lancinante rumeur du monde pour en extraire et rendre sonore ce qui, autrement, s'écoulait imperceptiblement. «Un matériau très complexe est chargé de rendre appréciables et perceptibles des forces d'une autre nature, durée, temps, intensité, silence, qui ne sont pas sonores en elles-mêmes 32 ".

Le temps pulsé et la musique formelle ont été pendant très longtemps les dispositifs qui permettaient de rendre sonores certaines forces, de dégager un filet de temps non pulsé. II n'y a, il ne peut qu'y avoir des mixtes de temps pulsé / non pulsé. Mais tout est question de proportion : tout dépend du coefficient de déterritorialisation de ces mixtes. Le temps pulsé fait territoire, mais comme tout territoire, il est travaillé de l'intérieur par des tendances qui cherchent à en sortir, temps non pulsé33. Le temps pulsé fait territoire, cadastre rassurant de l'attente et de la mémoire, bulle réconfortante d'un habitus à toujours lui-même pareil. La déterritorialisation est la sortie du territoire, le "décodage» d'un code rassurant - inscription scellant la pérennité de l'identique, marquage enfant de la médiation qui peuple le monde des créatures de la récognition. La musique flottante bâtie sur la recherche active d'une trame sonore non pulsée augmente donc le coefficient de déterritorialisation, musique au delà du sédentarisme propre à la force striée du temps, musique qui enfourche des lignes vagabondes, musique qui nous met directement en contact avec ce qui, sans elle, resterait imperceptible. Au dedans du pulsé répond l'ouvert du non pulsé où se meuvent toutes les rencontres inattendues propres au dehors. Esthétique qui se pose contre un certain romantisme où le choc de la musique se limite aux pâmoisons des impressions qui résonnent dans le for intérieur d'un sujet souffrant, souffreteux, reprise de l'idée kafkaïenne : "antilyrisme: "Empoigner" le monde" pour le faire fuir, au lieu de le fuir lui-même, ou de le caresser ${ }^{34}$ ». 
Nous pouvons conclure cette partie en disant, (1-) que l'esthétique musicale deleuzienne a une visée critique qui renvoie la distinction temps pulsé / non pulsé au projet global d'une critique de la représentation en appelant à un nomadisme, et que (2-) cette visée critique n'est que le corollaire d'une visée existentielle et créatrice qui recherche une connexion directe avec les devenirs qui constituent la vie, élargit le champ de perception sonore dans le cas de la musique, cas particulier (mais privilégié) d'une recherche d'élargissement perceptif tout azimut - crée de nouveaux modes de vie ${ }^{35}$. Élargir la perception, ou comme le disait Nietzsche, atteindre à une "sensualité "intelligente" ( $\tilde{N}$ intelligentei Sinnlichkeit) ${ }^{36}$ ".

Maintenant que nous avons esquissé les grandes lignes de l'esthétique musicale deleuzienne, qui, rappelons le, contrairement à la peinture, ne fait l'objet d'aucun livre, laissons nous porter par cette petite ritournelle qui fait territoire, et qui par "sauts" successifs et retours sur elle-même finira par gonfler et fuir dans la grande ritournelle du monde : du monde de la musique passons à la musique comme monde.

L'harmonie traverse une crise à son tour, au profit d'un chromatisme non élargi, d'une émancipation de la dissonance ou d'accords non résolus, non rapportés à une tonalité

Le Pli, p. 112

De l'harmonie au chromatisme élargi, passage de Leibniz à ce que Deleuze appelle le néo-leibnizianisme contemporain : «il semble difficile de rester insensible à l'ensemble de l'harmonie leibnizienne qui se fonde au même moment dans la musique baroque 37 ». Deleuze signale encore une fois qu'il ne s'agit pas ici de métaphore : il y a bien un rapport, un écho, une résonance, entre la musique baroque, sa conception de l'harmonie et la philosophie de Leibniz, symphonie concertante d'une harmonie préétablie. Lecture étonnante, mais dont l'exposé nous amènerait trop loin de ce que nous proposons de développer dans cet article, nous préférons nous concentrer sur le «chromatisme élargi" proposé et déployé par Deleuze lui-même, en nous attardant sur le concept de ritournelle : «'univers, le cosmos est fait de ritournelles : la question de la musique est celle d'une puissance de déterritorialisation qui traverse la nature». Ici, Deleuze, flanqué de son compère à la créativité débridée, Guattari, exposera l'étonnante singularité de sa philosophie dans le paysage de la pensée du XXe siècle en se faisant physicien, physique toute tissée 
d'une subtile métaphysique, rejoignant dans cet espace achronologique qu'est la philosophie qui se crée, le jeu du monde des présocratiques.

Ritorno, ritornare, volute d'une petite musique qui se répète; comme tous les concepts deleuziens, la ritournelle ne se laisse pas appréhender dans le carcan d'une définition : il faut donc suivre son vagabondage, sa variation continue pour comprendre sa logique. Nous suivrons donc le cheminement conceptuel lié à la ritournelle, "chant de la terre", fil conducteur d'une étonnante physique philosophique, et nous dirons finalement quelques mots du rapport plus particulier de la ritournelle à la musique.

Le rapport de la ritournelle à la terre se joue en trois moments : 1en elle s'esquisse la promesse d'une terre, d'un territoire au sein des vents furieux du chaos, un centre se crée, 2- elle constitue une terre contre les forces du chaos, un cercle se forme, 3- elle ouvre le cercle, pousse vers une sortie du territoire pour joindre un ailleurs qui n'est pas hors monde ni chaos, mais alliance avec des devenirs cosmiques en lesquels nous nous donnons au monde, nous confondant ainsi d'autant plus avec lui (cas idéal d'un devenirimperceptible, sortir d'un territoire signifiant souvent aussi : retomber dans un autre).

1- Infra-agencement. Du chaos, qui n'est pas désordre mais nébuleuse de forces non liées où tout se dissout dans une vitesse infinie ${ }^{38}$, naissent les milieux et les rythmes. "Chaque milieu est vibratoire, c'est-à-dire un bloc d'espace-temps constitué par la répétition périodique de la composante ${ }^{39}$ ». C'est le premier niveau de la ritournelle : un enfant, seul dans le noir, fredonne pour se rassurer, tra, la, la... Chaque milieu est codé, c'est-à-dire est constitué de sa propre répétition et est en permanence en état de transcodage, aptitude des milieux à passer les uns dans les autres. Car si les milieux sont en danger de se dissoudre dans le chaos, le rythme, l'entre-deux -, les fait fuir les uns dans les autres dans une grande sarabande les protégeant de toute dissolution. C'est en effet le rythme que les milieux opposent au chaos, rythme qu'il ne faut surtout pas confondre avec la froide mécanique de la mesure et de la cadence régulière ou irrégulière, répétition subordonnée à une métrique donnée a priori, mais qu'il faut plutôt concevoir comme l'Inégal ou l'Incommensurable, répétition faisant toujours passer un code dans un autre, distillant perpétuellement une différence (le rythme n'est pas sur le même plan que ce qu'il rythme). Le rythme n'est pas une 
métrique reproduisant l'égal ou l'inégal, selon un modèle posé à l'avance : c'est la répétition qui produit la différence ${ }^{40}$.

Donc à ce premier niveau, la ritournelle pointe et organise avec les milieux et les rythmes arrachés au chaos le seuil d'agencement territorial : un milieu passe dans un autre, ça coule, c'est le soubassement directionnel du monde, tout fuit en extension. De ces petites ritournelles un frêle mais déjà solide filet de consistance se déploie, ribambelle rythmée de milieux, de la mer au rivage, du rivage à la plaine, de la plaine à la forêt (et déjà la mer est une multitude de milieux, le poisson est un composé de milieux, etc.).

2- Intra-agencement. Mais ces petites ritournelles de milieux gonflent et un niveau de consistance supérieur est atteint avec le territoire. "Le territoire est le produit d'une territorialisation des milieux et des rythmes ${ }^{41}$ ». La ritournelle fait territoire en tant qu'elle emprunte à tous les milieux pour leur arracher des composantes, auto-position d'un tenir-ensemble d'hétérogènes. "ll y a territoire dès qu'il y a expressivité du rythme ${ }^{42}$ ». C'est l'expressivité qui fait territoire, non pas l'agressivité comme l'affirme Lorenz. Les composantes de milieux, coulant en extension, sélectionnées et assemblées dans ce va-et-vient de la ritournelle, perdent leur caractère directionnel pour devenir dimensionnelles : intensité ou expression. Sélectionnées dans l'élan d'une ritournelle, des composantes de milieu deviennent qualités et propriétés. Dans le va-et-vient des ritournelles, des qualités pures du milieu cessent d'être seulement fonctionnelles pour devenir des traits d'expressions, "sensibilia": création des blocs de sensations. La marque fait territoire, le marquage fait expression, l'urine peut couler sur les milieux ou devenir excrément territorial en bornant un domaine, cercle expressif d'un territoire. "La territorialisation est l'acte du rythme devenu expressif, ou des composantes de milieux devenues qualitatives. Le marquage d'un territoire est dimensionnel, mais ce n'est pas une mesure, c'est un rythme ${ }^{43}$ ". Le rythme, qui dans un premier temps était ce qui faisait passer les milieux les uns dans les autres, pose, en devenant expressif, un nouveau plan distinct des milieux : le territoire, résultat du processus rythmique et sélectif de territorialisation. Le facteur $T$, facteur territorialisant, est en effet «le devenir-expressif du rythme ou de la mélodie, c'est-à-dire l'émergence de qualités propres (couleur, odeur, son, silhouette....) $)^{44}$ ».

Cette expression est le symptôme de l'art, qui loin d'être le privilège de l'homme, apparaît dès qu'il y a affiche, pancarte, sorte de 
leitmotiv qui crie «à moi!» ou «le mien!». L'être-au-monde du vivant est artistique, l'expression est première : «l'expressif est premier par rapport au possessif, les qualités expressives, ou matières d'expression sont forcément appropriatives, et constituent un avoir plus profond que l'être 45 ". L'avoir étant plus profond que l'être, la signature, loin d'être la «marque constituée» d'un sujet est la «marque constituante" d'un domaine. À la clairière et au domaine du dasein comme ouverture et attente anticipante d'une venue de l'Être, Deleuze oppose à Heidegger le primat vital d'une création expressive fondamentale qui joint le vivant au monde, domaine où la vie gagne en intensité, passion et mystère de l'avoir qui nous donne au monde. Le constructivisme méthodique prôné pour la création de concept est rejoué ici au niveau existentiel, commençant à un niveau infra-humain, et posant les mots d'ordre : toujours plus d'expression, grandeur de l'artificialité - arcane de la nature, devenir-imperceptible qui n'est pas une fuite du monde, malheur de l'anachorète.

"La ritournelle, c'est le rythme et la mélodie territorialisés, parce que devenus expressifs - et devenus expressifs parce que territorialisants ${ }^{4 \%}$ ». L'expression est cause d'elle-même : à chaque fois qu'il y a expression, il y a auto-mouvement de qualités expressives. Les émotions subjectives sont des effets et non des expressions, tout comme les impulsions de milieu, telles les hormones qui colorent les organes de tel poisson ${ }^{47}$. Le poisson de corail, lui, par contre, marque un territoire avec les couleurs de ses organes, les couleurs deviennent ainsi expressives, elles sont auto-objectives "c'est-à-dire trouvent une auto-objectivité dans le territoire qu'elles tracent 48 ». Cette "fondation» spontanée qui se fonde par elle-même, stabilité instable, ou plutôt cette consistance qui "prend" d'elle-même, c'est fondamentalement le mouvement même de l'immanence, auto-mouvement, pirouette ontologique, «le revenir, c'est l'immanence attribuée à elle-même ${ }^{49}$ ". Nous retrouvons à ce niveau de consistance, celui de la ritournelle territorialisante, un goût d'immanence qui laisse déjà deviner LE plan d'immanence de la grande ritournelle 50 .

La ritournelle pose donc des milieux arrachés au chaos, les fait passer les uns dans les autres grâce au rythme, puis en sélectionnant des composantes de milieux qu'elle exprime, fait territoire, intensité auto-posée, marque, signature. Mais l'intensité du territoire, dans la mouvance perpétuelle des ritournelles, ne reste pas au stade de la signature : l'intensité - le territoire - s'étoffe en devenant style : «les 
qualités expressives ou matières d'expression entrent, les unes avec les autres, dans des rapports mobiles qui vont "exprimer» le rapport du territoire qu'elles tracent avec le milieu intérieur des impulsions et avec le milieu extérieur des circonstances ${ }^{51}$ ». En entrant en rapport les unes avec les autres, les qualités expressives forment des motifs territoriaux, motifs qui sont constitués des rapports internes des qualités expressives, ainsi que des jeux et entrelacs rythmiques des impulsions internes au milieu. On appelle aussi ces motifs territoriaux des visages ou personnages rythmiques ${ }^{52}$. La ritournelle donne en effet naissance à des personnages rythmiques qui ne sont pas des êtres auxquels serait attribué un rythme : c'est le rythme lui-même qui se singularise, qui devient visage, personnage. Ces rythmes qui prennent consistance pour eux-mêmes, ces motifs territoriaux s'étoffent par ailleurs dans des contre-points territoriaux, c'est-à-dire dans d'autres rapports internes des qualités expressives : "cette fois, c'est la manière dont elles constituent dans le territoire des points qui prennent en contre-point les circonstances du milieu externe ${ }^{53}$ ». On appelle aussi ces contre-points rythmiques paysages mélodiques. Tout comme le rythme s'autonomise, le paysage mélodique n'est pas un paysage auquel s'adjoint une mélodie, mais bien une mélodie qui devient elle-même paysage. Alors le rythme, la mélodie peuvent augmenter, diminuer, selon ce que captent les soubresauts des agrégats fluents, volutes d'hétérogènes emportées par telle ou telle petite ritournelle particulière, l'important est que les personnages rythmiques et les paysages mélodiques instaurent des rapports qui restent constants : autonomie du rapport. À ce niveau de consistance, le territoire n'est plus simple marquage, mais développe un style, consistance supérieure, dans un opéra machinique ${ }^{54}$. Machinisme qui n'est pas un linéarisme causal, trivial enchaînement de cause à effet, mais un complexe de rapports mobiles de qualités expressives les unes avec les autres, symphonie de la Nature. Nouvelle définition, donc, de la ritournelle : «tout ensemble de matières d'expression qui trace un territoire, et qui se développe en motifs territoriaux, en paysages territoriaux ${ }^{55}$ ».

Il faut noter ici que l'écueil structuraliste est subtilement évité : en effet le monde est constitué de lignes ${ }^{56}$, faites de rhizomes, système fluide de connexions à plat, en surface, destituant l'idée de structure et son empire qui s'étale en hauteur et profondeur, organicisme d'un système de points reliés. Entre les lignes se produisent des rencontres, fourmillent les divergences, se créent des blocages, se jouent 
des accords, éclate ce qui fulgure, se déploient des résonances, effets d'une communication entre intensités disparates (nouveau niveau d'intensité). De Schonberg à Stockhausen, de Lévi-Strauss à Deleuze, les points capitons des séries sautent, laissant couler plus librement les séries. Notons aussi que l'auto-position de l'expression et l'autonomie des rapports des personnages rythmiques et des paysages mélodiques évitent l'identification et la récognition trop simple des objets, sujets et milieux selon les schémas du sens commun, aussi qu'une trop simple idée des déterminismes de milieux.

3- inter-agencement : «Une composante territoriale ou territorialisée peut se mettre à bourgeonner, à produire : c'est tellement le cas de la ritournelle qu'il faut peut-être appeler ritournelle tout ce qui est dans ce cas ${ }^{57}$ ». Le territoire fait agencement, en tant que la ritournelle fait tenir-ensemble des éléments hétérogènes, mais cette stabilité vibratoire, rythmique, est travaillée de l'intérieur par des tendances qui poussent à la sortie du territoire : "c'est que l'agencement territorial n'est pas séparable des lignes ou coefficients de déterritorialisation, des passages et des relais vers d'autres agencements 58 ». "On» quitte le territoire : déterritorialisation. Le bloc d'expression rythmé qu'est le territoire est travaillé en permanence par des tendances de déterritorialisation qui tendent à l'ouvrir à d'autres rythmes, d'autres territoires, lignes de fuite d'une genèse perpétuelle. Le territoire est de passage : les lignes de déterritorialisation ouvrent l'agencement territorial sur d'autres agencements, et les font passer les uns dans les autres. La ritournelle atteint donc cette puissance supérieure de déterritorialisation qui empêche le territoire de se fermer, de tomber dans une cadence trop dure, une petite rengaine. Sortir du territoire, c'est en un sens "défaire» ce qui s'était construit. La ritournelle à ce niveau devient ce qui engendre et ce qui détruit, devenir perpétuel territorialisation /déterritorialisation 59 .

On aura compris la visée critique de ce concept de déterritorialisation : le territoire peut être physique, mais est aussi bien pratique, éthique, esthétique, politique, mental. En lui est codé un contenu, c'est-à-dire est donnée une forme, une signification à un flux, une multiplicité, une intensité. La déterritorialisation, puissance de "déblocage" se trouvant toujours, du moins à l'état latent, dans tout territoire - ça fuit constamment de partout, malgré les blocages -, sera activement recherchée, activée et construite, pour laisser filer sur une ligne de fuite, ligne de vie, des contenus pris dans toute sorte de formes. Sortir du territoire : "c'est l'opération ligne de fuite ${ }^{60}$ ". Mais, 
encore un fois, il ne s'agit pas d'une fuite hors du monde, d'un amour immodéré pour le néant : il s'agit d'une fuite en avant, du chaos au cosmos en passant par le territoire, devenirs successifs de la ritournelle dans des prises de consistances successives, affirmant le tout du monde dans des niveaux, des plateaux d'intensité augmentant toujours en puissance : «il n'y a pas le Temps comme forme pure a priori, mais la ritournelle est la forme a priori du temps, qui fabrique à chaque fois des temps différents 61 ».

De ritournelles en ritournelles, il s'agit toujours d'élargir la perception, d'en arriver à mieux embrasser le monde par sauts successifs, en créant les formes les plus fluides possibles comme tremplin pour des seuils d'intensité supérieurs, perpétuel dynamisme de l'accroissement de la sensation (volonté de puissance comme augmentation de la force qui, comme le remarque Deleuze, est pour Nietzsche le pouvoir d'être affecté).

La ritournelle, motif, air qui se répète dans le langage courant, devient sous la plume des auteurs de Mille plateaux le processus même du devenir, avec son entrelacs perpétuel de formes qui se font et se défont : déjà chez l'animal, se fait et se défait le territoire dans des ritournelles "gestuelles, posturales, chromatiques, visuelles". Mais, nous dit-on, il y a privilège de la ritournelle sonore en ce qui a trait à son pouvoir de déterritorialisation : «il semble que le son, en se déterritorialisant, s'affine de plus en plus, se spécifie et devienne autonome. (...) le son nous envahit, nous pousse, nous entraîne, nous traverse 62 ". Le son tend plus que toute autre sensation à s'autonomiser (la couleur, par exemple, «tient» plus au territoire) : ce qui donne la belle définition de la musique comme ritournelle déterritorialisée ${ }^{63}$. Car la ritournelle sonore fait spontanément territoire : "ritournelle d'enfance ou d'oiseau, chant folklorique, chanson à boire, valse de Vienne, clochette à vache, la musique se sert de tout et emporte tout64». La musique comme puissance de consolidation et de déterritorialisation supérieure : nous pouvons maintenant exposer un parallèle entre la physique du monde vivant et la prise de consistance musicale : 1 - a) un territoire se crée en arrachant un bloc de sensation, en exprimant des qualités d'un milieu coulant en extension, b) à partir de la rumeur du monde, avec ses bruits, ses sons, dirigés en tout sens, se créent des ritournelles sonores posant territoires (berceuse, marche militaire, comptine, thèmes musicaux et formes de compositions devenues de plates habitudes...) 2- a) des forces de déterritorialisation travaillent le territoire en l'ouvrant vers un 
ailleurs, ouverture sur le cosmos comme affirmation-sensation du monde supérieur, b) la musique déterritorialise les petites ritournelles qui font territoire pour les emporter dans un devenir musical, pur devenir d'un filet musical qui rend sonores des forces sans lui inaudibles.

"Un seul organisme, possédant plusieurs climax, plusieurs couches à la fois et se développant à différentes vitesses, différentes densités" : on pourrait croire, ici, à une définition de l' tre univoque deleuzien, plan d'immanence sur lequel se développent et s'évanouissent des plateaux d'intensité, bien qu'il s'agisse de Stockhausen décrivant deux de ses œuvres, Punkte et Gruppen65. Cet air de famille entre la pensée d'un musicien et celle de Deleuze n'est pas étonnant, quand on sait que notre philosophe s'est inspiré plus que tout autre de la musique, lui donnant une esthétique correspondant à l'actualité de sa création, et s'inspirant de cette création pour sa propre philosophie. Car pour Deleuze, la philosophie ne doit pas faire discours sur quelque chose, mais doit participer au mouvement de cette chose selon ses propres moyens. Nous avons vu en première partie que son esthétique musicale s'insérait dans une visée critique contre le système de la représentation, prélude à une visée existentielle vitaliste, ethos du vagabondage qui «empoigne le monde» dans un processus continu d'élargissement perceptif. Pour participer selon ses propres moyens au mouvement de quelque chose, il faut d'abord dégager ce mouvement. Dans un deuxième temps, nous avons vu comment la musique, art non représentatif par excellence, lui fournit un riche matériel pour sa création conceptuelle, notamment dans le cadre de sa physique philosophique : problème de la consistance dans un monde de flux vagabonds, problématique conjointe à la philosophie et à la musique d'aujourd'hui. Deleuze définit comme suit les problématiques communes de la musique et de la philosophie à l'âge moderne par rapport à l'âge classique et l'âge romantique : "Le rapport essentiel n'est plus matières-formes (ou substances-attributs); mais il n'est pas davantage dans le développement continu de la forme et la variation continue de la matière. II se présente ici comme un rapport direct matériau-forces. Le matériau, c'est une matière molécularisée, et qui doit à ce titre "capter» des forces, lesquelles ne peuvent plus être que des forces du Cosmos66». Idée de Cosmos prise à Stockhausen dont l'idée de l'art comme "genèse permanente ${ }^{67}$ " trouve un écho singulier dans l'idée deleuzienne de la philosophie comme art de la recherche de la genèse des choses et de 
la pensée - privilégier le processus plutôt que le résultat -, tout comme l'appel au constructivisme conceptuel pour accéder à un concentré des forces du chaos rappelle étonnamment cette idée boulezienne d'un constructivisme pour atteindre à une ligne vagabonde musicale au temps non pulsé : "on ne peut trouver la liberté que par la discipline !68». (En ce sens, malgré deux ethos complètement différents, ne pourrait-on pas rapprocher Cage et Heidegger - critique radicale de la tradition, de la détermination musicale pour l'un, de la détermination du système métaphysique de la représentation pour l'autre : recherches d'espaces ouverts pour une attente contemplative?...). Peu de philosophes, en tout cas, auront eu des rapports si étroits avec la musique, et à coup sûr, aucun ne se sera abreuvé comme Deleuze à l'extraordinaire créativité de la musique contemporaine.

\author{
Vincent Jacques \\ EHESS, Paris
}

1. Cf. "Sur la volonté de puissance et l'éternel retour», Gilles Deleuze , île déserte et autres textes (1953-1974), Paris, Éditions de Minuit, 2002.

2. Contrée deleuzienne qui est aussi en partie la création de Félix Guattari, coauteur de certains ouvrages, notamment de Mille Plateaux. Pour ne pas alourdir le texte nous écrirons indistinctement Deleuze, qu'il s'agisse aussi bien de Deleuze seul que de Deleuze-Guattari. De toute façon, comme l'écrivaient justement les deux auteurs, "Je est légion", et rien n'est plus étranger à une pensée nomade qu'une répartition des tâches, des réalisations et des gloires respectives. (Pour plus de précisions, voir la bibliographie).

3. Omnis negatio est determinatio démontrait Spinoza, CEuvres complètes, Paris, Gallimard, 1954, lettre 50. Toute détermination est négation : on peut dire que Deleuze reprend cette intuition spinoziste, point de fuite vers lequel tend toute sa philosophie. Les formes, la médiation, la représentation, CEdipe, sont autant de blocages négatifs qui obstruent le libre cours de la vie. D'où l'importance de la déterritorialisation, échappée hors de tout domaine, routine, rengaine du quotidien et affres du sens commun. Mais nous disons bien intuition, car en effet, comme nous le verrons plus loin, un minimum de formes est nécessaire pour que la vie soit "viable", et même, certains processus de déterminations sont nécessaires à une intensification des devenirs vitaux. Cette idée que toute détermination est une négation, est mise en exergue par Gebhardt, pour qui el'affranchissement de la forme" (Entformheit) est ce qui ferait de Spinoza un auteur baroque; on peut dire aussi que cet Entformheit est bien une des préoccupations majeures de Deleuze. C. Gebhardt, Spinoza, judaïsme et baroque (Groupe de recherche spinoziste no 9), Paris, Presses de l'Université de Paris-Sorbonne, 2000. 


\section{Vincent Jacques}

4. Gilles Deleuze et Félix Guattari, Mille plateaux, Paris, Éditions de Minuit, 1980, p. 230.

5. Ibid., p. 369.

6. Notons au passage l'intérêt pour la multisensorialité de certains compositeurs contemporains, de la spatialité des recherches de Stockhausen aux entrelacs lumièrearchitecture de Xenakis.

7. Néologisme de A.Riegl, haptisch, à partir du verbe ámTw, toucher, repris librement par Deleuze dans son analyse des rapports œil/main dans l'acte de peindre: «on parlera d'haptique (...) quand la vue elle-même découvrira en soi une fonction de toucher qui lui est propre, et n'appartient qu'à elle, distincte de sa fonction optique" (p. 99).

8. M. Buydens, Sahara. L'esthétique de Gilles Deleuze, Paris, Vrin,1990, p. 12.

9. Deleuze et Guattari, Mille plateaux, p. 371.

10. M. Buydens, op. cit., p.146.

11. En vrac, l'idée claire et distincte, les lumières de la raison, le prophète : voyant, seer, seher, l'idée - eideon, image... Et les expressions populaires, ni vues, ni connues, lire dans quelqu'un comme dans un livre ouvert...

12. Deleuze et Guattari, Mille plateaux, p. 20.

13. Ibid., p. 371. Il suffit par exemple de penser à l'utilisation de l'opéra de Wagner par la propagande nazi.

14. Cf. P. Boulez, Penser la musique aujourd'hui, Denoël/Gonthier, 1963.

15. G. Deleuze, "Occuper sans compter; Boulez, Proust et le temps", Deux régimes de fous. Textes et entretiens 1975-1995, Paris, Éditions de Minuit, 2003, p. 274.

16. Ibid.

17. «ll n'y a pas de points ou de positions dans un rizome, comme on en trouve dans une structure, un arbre, une racine. II n'y a que des lignes" Deleuze et Guattari, Mille plateaux, p. 15.

18. A. Villani, "Géographie physique de Mille Plateaux», Critique, no 445, 1985, p. 339.

19. Malgré l'espace lisse crée par le capital : tout nomadisme n'est pas bon en soi.

20. Quid facti, quid juris. La vérification des faits, par exemple l'existence de cercles plus ou moins parfaits, n'empêche nullement qu'en droit, le cercle existe.

21. G. Deleuze, "Occuper sans compter...", p. 275.

22. Deleuze et Guattari, Mille Plateaux, p. 320. Distinction reprise aux Stoïciens, apparue dans Logique du sens. Selon Buydens, Deleuze aurait élaboré cette distinction à partir de l'intuition temporelle boulezienne (Buydens, op. cit., p.155).

23. G. Deleuze, “Occuper sans compter...», p. 276.

24. Ibid., p. 278.

25. Stockhausen parle de "plaques sonores", "d'événements sonores", de "densités", de la nécessité "de créer des processus à l'image de cette nature en évolution et non une suite de "caractères", «j'aime qu'on voyage avec les sons, qu'on les poursuive". Cf. l'entrevue, "Karlheinz Stockhausen l'illusionniste ", Art press. Anatomie des cultures électroniques, hors série no 19, (1988), p.43-47.

26. "la perception d'une "phrase" musicale fait moins appel à une mémoire, même du type réminiscence, qu'à une extension ou contraction de la perception du type rencontre» écrit Deleuze a propos de «la haine de mémoire» propre à Boulez. Mille Plateaux, p. 364. 
27. L'évidente banalité de la deuxième assertion n'est là que pour mettre en exergue la première, montrant le refus radical de tout évolutionnisme chez Deleuze, ce qui peut sembler banal dans le domaine des arts, mais n'est toujours pas admis dans le domaine philosophique. II n'y a pas de progrès de la connaissance, seulement les problèmes que doit affronter la pensée changent, et l'histoire de la philosophie ne vaut rien si elle n'est capable d'articuler ses travaux sur ce qui fait problème aujourd'hui, si elle n'est capable d'actualiser une virtualité «éternelle" de la philosophie créée à des époques différentes, d'insuffler vie pour faire danser des philosophes trop souvent réduits à l'état de statue.

28. "c'est comme si l'âge des insectes avait relayé le règne des oiseaux, avec des vibrations, des stridulations, des crissements, des bourdonnements, des claquements, des grattages, des frottements beaucoup plus moléculaires". Mille Plateaux, p. 379. Ondes Martenot, synthétiseurs, en produisant directement la vibration, sont des instruments pour l'ère musicale du temps non pulsé (les ondes, les intensités, les densités sont rebelles à toute métrique).

29. G. Deleuze, "Occuper sans compter...", p. 276.

30. $\propto$ On prétend ouvrir la musique à tous les événements, à toutes les irruptions, mais, ce qu'on reproduit finalement, c'est le brouillage qui empêche tout événement. (...) Un matériau trop riche est un matériau qui reste trop "territorialisé", sur les sources des bruits, sur la nature des objets... (même le piano préparé de Cage)". Mille Plateaux, p. 424.

31. "Donner consistance sans rien perdre de l'infini» G. Deleuze et F. Guattari, Qu'estce que la philosophie?, Paris, Éditions de Minuit, 1991, p. 45. II s'agit de construire des plans qui coupent le chaos et en conservent le mouvement dans des entités Chaoïdes - (et non chaotiques, il s'agit de donner un peu de consistance au chaos) construites par la science, l'art et la philosophie.

32. "Le temps musical", conférence donnée à l'IRCAM en 1978 où sont identifiés quelques "êtres musicaux» : paysage sonore, couleur audible, personnage rythmique (www.webdeleuze.com). Deleuze dit aussi «identifier la variation» («Occuper sans comptern, p. 277) : de là sa fascination pour la musique répétitive de Steve Reich, variation continue, jeux sonores de la répétition créant différence.

33. "le cadastre est une pulsation du temps", "Le temps musical».

34. G. Deleuze et F. Guattari, Kafka. Pour une littérature mineure, Paris, Éditions de Minuit, 1975, p. 109.

35. "penser signifierait ceci : découvrir, inventer de nouvelles possibilités de vie» écrit Deleuze dans son Nietzsche et la philosophie, Paris, Éditions de Minuit, 1965, p.115, assertion qu'il reprend bien sûr à son compte et qu'il faut mettre en parallèle avec les injonctions à élargir la perception, un fois dit que l'art, celui qui vaut quelque chose, pense aussi et que la philosophie est un art : «nous les artistes = nous les chercheurs de connaissances ou de vérité = nous les inventeurs de nouvelles possibilités de vie», p. 117.

36. F. Nietzsche, «Fragment posthume, printemps 1888, 14», Fragments posthumes XIV, Paris, Gallimard, 1977, p. 117.

37. G. Deleuze, Le Pli. Leibniz et le baroque, Paris, Éditions de Minuit, 1988, p. 186.

38. «On définit le chaos moins par son désordre que par la vitesse infinie avec laquelle se dissipe toute forme qui s'y ébauche. C'est un vide qui n'est pas un néant, mais un virtuel, contenant toutes les particules possibles et tirant toutes les formes possibles qui surgissent pour disparaître aussitôt, sans consistance ni référence, sans conséquence. C'est une vitesse infinie de naissance et d'évanouissement.» Qu'estce que la philosophie?, p. 111-112. 


\section{Vincent Jacques}

39. Deleuze et Guattari, Mille Plateaux, p. 380.

40. Une des thèses principales de Différence et répétition.

41. Mille Plateaux, p. 386.

42. Ibid., p. 387.

43. Ibid., p. 388.

44. Ibid.

45. Ibid., p. 389.

46. Ibid.

47. Cette idée d'une distinction entre des animaux artistes, car posant territoire, et animaux non artistes, renvoie à l'intuition de Messiaen «n'y a-t-il pas des oiseaux musiciens, comme le pense Messiaen, par différence avec des oiseaux non musiciens?» Ibid., p. 370.

48. Ibid., p. 390.

49. G. Deleuze, Qu'est-ce que la philosophie?, p. 40.

50. De ritournelles en ritournelles, augmentation des cercles de puissances, jusqu'à l'idée de la ritournelle de toutes les ritournelles, l'éternel retour (qu'il ne faut pas confondre avec le banal cycle des saisons), aller-retour du plan d'immanence : "ce qui définit le mouvement infini, c'est un aller-retour. Le plan a deux faces. Le mouvement infini est double et il n'y a qu'un pli de l'un à l'autre" (Qu'est-ce que la philosophie?, p. 40). Aussi, la définition de Mille Plateaux de l'éternel retour comme ritournelle : «qui capture les forces muettes et impensables du Cosmos», p. 423.

51. Mille Plateaux, p. 390 . Nous soulignons.

52. Deleuze emprunte la notion de "personnage rythmique» à Messiaen. cf. Mille Plateaux, p. 392. On peut souligner aussi qu'ici le leitmotiv cesse d'être «pancarte», pour acquérir une autonomie qui lui est propre : une idée, cette fois, empruntée à P'analyse de Boulez du Ring. cf. Penser la musique aujourd'hui.

53. Mille Plateaux, p. 390.

54. Soit un oiseau des forêts humides d'Australie, le Scenopoiëtes dentisoris, qui chaque matin coupe les feuilles de son arbre, les retourne sur le sol pour que leur face la plus pâle contraste avec la terre plus obscure, pour ensuite chanter un savant contrepoint composé de ses propres notes et de celles d'oiseaux d'espèces différentes, tout en exhibant les plumes jaunes du dessous de son bec. Cf. Mille Plateaux, p. 387-389, 408, 415 (et sur l'autonomie du rapport on peut aussi penser aux «formules" de Stockhausen...)

55. Mille Plateaux, p. 393.

56. «le rhizome n'est fait que de lignes : lignes de segmentarité, de stratification, comme dimensions, mais aussi lignes de fuite ou de déterritorialisation comme dimension maximale d'après laquelle, en la suivant, la multiplicité se métamorphose en changeant de nature" Ibid., p. 32

57. Ibid., p .401.

58. Ibid., p. 410.

59. L'enchainement conceptuel est plus complexe, il a aussi des reterritorialisations, des déterritorialisations absolues, plusieurs sens à ces déterritorialisations absolues, mais l'exposé de toutes ces nuances nous amènerait hors du cadre que nous nous sommes fixé pour cet article...

60. Ibid., p. 634. 
61. Ibid., p. 431 , et «la ritournelle a aussi une fonction catalytique : non seulement augmenter les vitesses des échanges et réactions de ce qui l'entoure, mais assurer des interactions indirectes entre des éléments dénués d'affinités dites naturelles, et former par là des masses organisées" Ibid., p. 430.

62. Ibid., p. 429.

63. «La musique est précisément l'aventure d'une ritournelle : la manière dont la musique retombe en ritoumelle (...); la manière dont elle s'empare de la ritournelle, la rend de plus en plus sobre, quelques notes, pour l'emporter sur une ligne créatrice d'autant plus riche, dont on ne voit ni l'origine ni le bout..." Ibid., p. 370-371.

64. Ibid., p. 431. Nous soulignons.

65. K. Stockhausen in Art press. Anatomie des cultures électroniques, p. 46.

66. Ibid., p. 422.

67. Cité dans P. Boulez, Par volonté et par hasard, Paris, Éditions du Seuil,1975, p. 75. Autre résonance, la fascination de Stockhausen pour les formules simples, libérant de plus ou moins grandes plages musicales, de Mantra à la démesure de Licht, et la fascination de Deleuze pour le modèle de l'œuf, virtuel qui actualise des plages d'espaces-temps selon des vitesses et des rythmes différentiels (voir G. Deleuze, Différence et répétition, Paris, PUF, 1968, p. 276-279 : «le monde entier est un œuf»).

68. P. Boulez, Penser la musique aujourd'hui, p. 9. 